\title{
Towards Fabrication of Planar Magnetoelectric Devices: Coil-Free Excitation of Ferromagnet-Piezoelectric Heterostructures
}

\author{
Dmitri Burdin ${ }^{1}$, Dmitri Chashin ${ }^{1}$, Leonid Fetisov ${ }^{1}{ }^{\circledR}$, Dmitri Saveliev ${ }^{1} \oplus$, Nikolai Ekonomov ${ }^{1}$, \\ Melvin Vopson ${ }^{2}$ (D) and Yuri Fetisov ${ }^{1, *(D)}$ \\ 1 Research and Education Centre "Magnetoelectric Materials and Devices", MIREA-Russian Technological \\ University, 119454 Moscow, Russia; phantastic@mail.ru (D.B.); chashin@mirea.ru (D.C.); \\ fetisovl@yandex.ru (L.F.); dimsav94@gmail.com (D.S.); economov@list.ru (N.E.) \\ 2 School of Mathematics and Physics, University of Portsmouth, Portsmouth PO1 SHF, UK; \\ melvin.vopson@port.ac.uk \\ * Correspondence: fetisov@mirea.ru
}

\section{check for}

updates

Citation: Burdin, D.; Chashin, D.; Fetisov, L.; Saveliev, D.; Ekonomov, N.; Vopson, M.; Fetisov, Y. Towards Fabrication of Planar Magnetoelectric Devices: Coil-Free Excitation of Ferromagnet-Piezoelectric Heterostructures. Actuators 2021, 10, 294. https://doi.org/10.3390/ act10110294

Academic Editors: Carmine Stefano Clemente and Daniele Davino

Received: 10 October 2021

Accepted: 2 November 2021

Published: 4 November 2021

Publisher's Note: MDPI stays neutral with regard to jurisdictional claims in published maps and institutional affiliations.

Copyright: (c) 2021 by the authors. Licensee MDPI, Basel, Switzerland. This article is an open access article distributed under the terms and conditions of the Creative Commons Attribution (CC BY) license (https:// creativecommons.org/licenses/by/ $4.0 /)$.
Abstract: Magnetoelectric (ME) effects in composite ferromagnet-piezoelectric (FM/PE) heterostructures realize the mutual transformation of alternating magnetic and electric fields, and are used to create magnetic field sensors, actuators, inductors, gyrators, and transformers. The ME effect in composite structures is excited by an alternating magnetic field, which is created using volumetric electromagnetic coils. The coil increases the size, limits the operating frequencies, and complicates the manufacture of devices. In this work, we propose to excite the ME effect in composite heterostructures using a new coil-free excitation system, similar to a "magnetic capacitor". The system consists of parallel electrodes integrated into the heterostructure, through which an alternating current flows. Modeling and measurements have shown that the excitation magnetic field is localized mainly between the electrodes of the magnetic capacitor and has a fairly uniform spatial distribution. Monolithic FM/PE heterostructures of various designs with FM layers of amorphous Metglas alloy or nickel-zinc ferrite and PE layers of lead zirconate titanate piezoceramic were fabricated and investigated. The magnitude of the ME effect in such structures is comparable to the magnitude of the ME effect in structures excited by volumetric coils. However, the low impedance of the coil-free excitation system makes it possible to increase the operating frequency, reducing the size of ME devices and the power consumption. The use of coil-free excitation opens up the possibility of creating planar ME devices, and accelerates their integration into modern electronics and microsystem technology.

Keywords: magnetoelectric effect; magnetostriction; piezoelectricity; heterostructure; magnetic capacitor; field sensor; actuator; transformer

\section{Introduction}

In recent years, much attention has been paid to the development of new devices of microsystem engineering and electronics, such as highly sensitive magnetic field sensors [1], magnetic actuators [2,3], inductors and gyrators [4,5], transformers [6,7], devices for processing microwave signals [8], low-frequency antennas [9], and data storage elements [10] based on magnetoelectric (ME) effects in composite ferromagnet/piezoelectric (FM/PE) heterostructures.

ME effects in planar heterostructures with FM and PE layers arise as a result of a combination of magnetostriction of the FM layer and piezoelectricity in the PE layer, due to mechanical coupling between the layers [11,12]. When the structure is exposed to an alternating magnetic field, the FM layer is deformed due to magnetostriction; this deformation is transmitted to the PE layer and it generates, due to the piezoelectric effect, an alternating electric voltage (direct ME effect). When an alternating electric field is applied to the structure, the PE layer is deformed due to the inverse piezoelectric effect; this deformation is transferred to the FM layer, which leads to a change, due to inverse 
magnetostriction, of the magnetic induction in this layer (converse ME effect). Thus, ME effects implement the mutual transformation of magnetic and electric fields in solid-state heterostructures. The conversion efficiency of the fields can be controlled by applying an additional dc magnetic or electric field to the structure. The efficiency of ME conversion increases sharply, due to an increase in deformations when the frequencies of the excitation fields coincide with the frequency of the acoustic resonances of the structure [13].

In devices using the direct ME effect (such as dc magnetic field sensors, actuators, gyrators, controlled transformers, etc.), an excitation magnetic field is usually created using a volumetric electromagnetic coil with an alternating current flowing through it. However, this method of excitation of the ME effect has a number of drawbacks. First, volumetric electromagnetic coils are large and not technologically advanced to manufacture. Second, the high inductance of the bulk coils limits the operating frequency, which does not allow reducing the size of the structures, since the acoustic resonance frequency is inversely proportional to the size of the structure. Third, the electromagnetic coils create magnetic interference in the surrounding space, which worsens the electromagnetic compatibility of ME devices. Therefore, a very urgent problem is the development of new methods for the excitation of the ME effect in planar FM/PE heterostructures.

In this work, to excite the ME effect in composite heterostructures, it is proposed to use an excitation system of the "magnetic capacitor" type. The system consists of parallel conducting electrodes located near the heterostructure or integrated into it, through which an alternating current flows. In the first part of the article, the characteristics of a magnetic capacitor are modeled. Then, the choice of materials for the layers of the structures is substantiated and the methods of measuring the characteristics of the ME effect in structures with coil-free excitation are described. After that, the results of experimental studies of monolithic planar ME heterostructures of various designs with an excitation system in the form of a magnetic capacitor are presented. In the conclusion, the main results of the work are summarized. To present a coherent story, the article contains new original results and some results previously published by the authors. In particular, for the first time, the spatial distributions of the excitation current and magnetic field in a "magnetic capacitor" system were calculated, the ME effects in monolithic structures of amorphous ferromagnet-piezoelectric and ferrite-piezoelectric with integrated excitation systems were observed and investigated, and the advantages of using coil-free excitation systems in ME devices were formulated.

\section{2. "Magnetic Capacitor" Excitation System}

The design of the excitation system of the "magnetic capacitor" type is shown schematically in Figure 1. It contains two conducting flat electrodes of length " $b$ " and width " $c$ " each, located in parallel at a distance " $a$ " from each other. The thickness of the electrodes is significantly smaller than all other dimensions, as well as the distance between them. The upper and lower electrodes are connected on the right side by a vertical section. The center of the Cartesian coordinate system is located at the center of the magnetic capacitor between the electrodes. On the left side, an alternating current with amplitude $I$ and frequency $f$ is supplied to the upper electrode through a wire. The current spreads over the width of the electrodes, as shown qualitatively by the arrows in Figure 1. In this case, the currents along the upper and lower electrodes flow towards each other.

Using the theorem on the circulation of the magnetic field $\oint h \cdot d l=\sum I$, it can be shown that a current $I$ flowing through an electrode of infinite length and width $\mathrm{c}$ creates a tangential field $h_{y}=I / 2 c$ near its surface. Two infinitely long flat electrodes, located at a small distance $a<<\mathrm{c}$ from each other, create a magnetic field in the surrounding space, which is a superposition of the fields generated by each electrode. The magnetic fields in the center between the electrodes are added up, resulting in a total field $h_{y} \approx I / c$, while the fields outside the electrodes cancel out, giving a total field close to zero. 


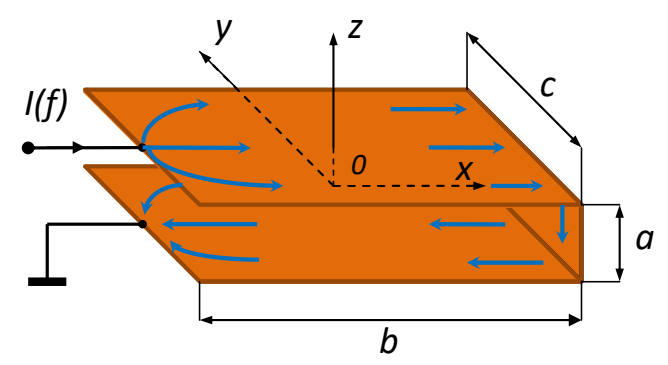

Figure 1. Schematic view of the "magnetic capacitor" excitation system. The arrows show the direction of the current in the electrodes.

Such a system can be considered as a "magnetic capacitor" - an analog of a flat "electric capacitor" [14]. It is known that in an "electric capacitor" the electric field $E$ is concentrated mainly between its plates, having a uniform spatial distribution and weakly extending beyond the capacitor. As it will be shown below, the magnetic field $h$ created by the "magnetic capacitor" is also concentrated mainly between its electrodes, it has a fairly uniform spatial distribution, and it extends only partially beyond its limits.

In a real excitation system shown in Figure 1, with two electrodes of finite length and the current being supplied from one side, the spatial distribution of the magnetic field, as can be expected, will be inhomogeneous both along the length and width of the system. The calculation of the real spatial distribution of currents and fields in such a system was carried out using the COMSOL Multiphysics package. For calculations, the dimensions of the system were $b=15 \mathrm{~mm}, c=10 \mathrm{~mm}, a=1 \mathrm{~mm}$, and the thickness of the copper electrode was $50 \mu \mathrm{m}$, corresponding to typical experimental conditions. The calculations were carried out for a range of frequencies of the excitation current, $f=0-100 \mathrm{MHz}$.

Figure 2 shows the calculated spatial distribution of the current density $j(x, y)$ in the upper electrode of the excitation system for a current amplitude $I=100 \mathrm{~mA}$ and a frequency $f=100 \mathrm{kHz}$. It can be seen that the current density is maximum near the point of current injection into the excitation system, and drops sharply with the distance from the injection point. At a distance of several millimeters from the injection point, the current distribution along the length and width of the electrode becomes practically uniform.

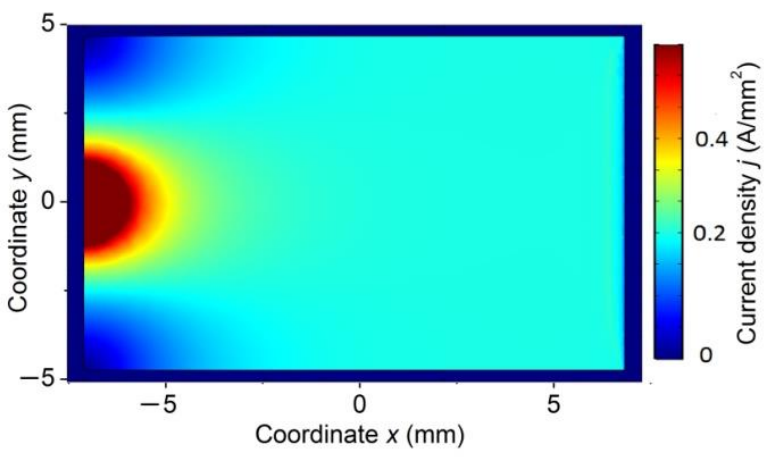

Figure 2. Spatial distribution of the electric current density in the upper electrode of "magnetic capacitor" for $I=100 \mathrm{~mA}$.

Figure 3 shows the calculated spatial distribution of the field component $h_{\mathrm{y}}$ in the $x-y$ plane between the electrodes of the magnetic capacitor at $z=0$. It can be seen that the field amplitude is also maximum near the current injection point and rapidly decreases with distance from the injection point. In the transverse direction along the $y$-axis, the field $h_{y}$ is maximal on the longitudinal axis of the excitation system and decreases towards its edges. 


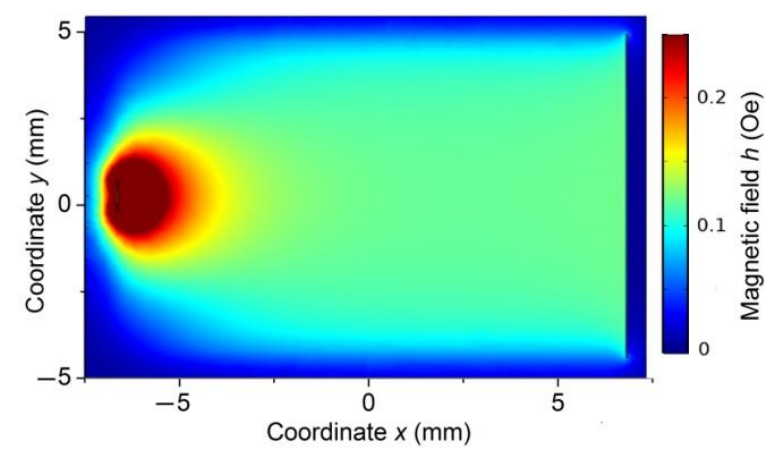

Figure 3. Spatial distribution of the magnetic field $h_{\mathrm{y}}$ inside the "magnetic capacitor" for $I=100 \mathrm{~mA}$ and $\mathrm{z}=0$.

Figures 4 and 5 show the quantitative characteristics of the spatial distribution of the field $h_{\mathrm{y}}$ between the electrodes at the amplitude of the excitation current $I=100 \mathrm{~mA}$ and a frequency of $100 \mathrm{kHz}$. It can be seen that on the longitudinal axis of the system $(y=0)$, the field decreases from $\sim 0.8$ Oe near the current injection point to 0.12 Oe at a distance of $\sim 3 \mathrm{~mm}$ from the injection point, and then practically does not change when moving along the axis to the end of the electrode. Outside the excitation system, the field decreases by 1 order of magnitude as the distance from the injection point is $\sim 2 \mathrm{~mm}$, and by $\sim 3$ orders of magnitude immediately behind the closed end of the system. In the transverse direction along the $y$-axis, the field between the electrodes is mostly inhomogeneous near the point of current injection into the system $(x=-b / 2)$, becomes more uniform in the middle part of the system $(x=0 \mathrm{~mm})$, and then, when moving to the end of the electrode, the field distribution is practically not changed. Outside the system, the field in the transverse direction decreases by 1 order of magnitude, as the distance from the edges of the electrodes is $\sim 1.7 \mathrm{~mm}$.

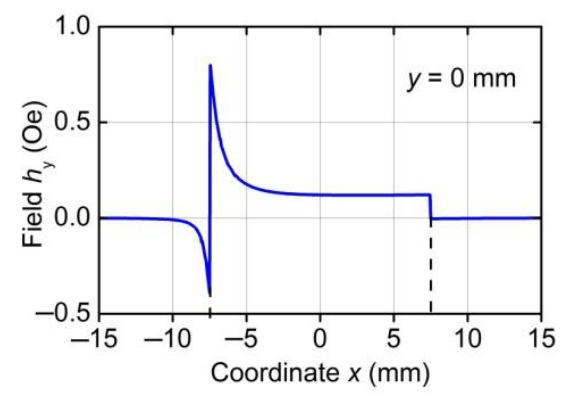

Figure 4. Distribution of the magnetic field $h_{\mathrm{y}}$ along the length inside the "magnetic capacitor" for $I=100 \mathrm{~mA}$ and $z=0$.

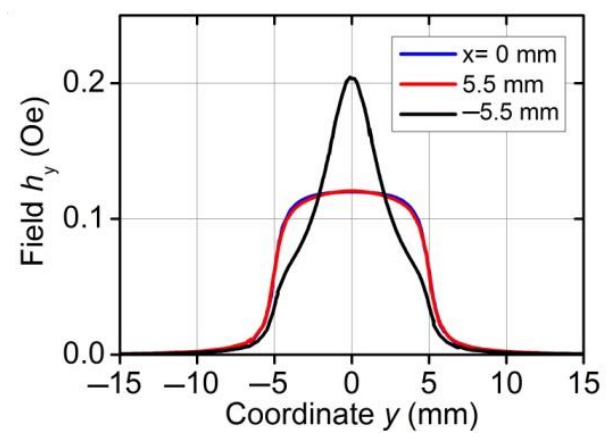

Figure 5. Distribution of the magnetic field $h_{\mathrm{y}}$ along the width inside the "magnetic capacitor" for $I=100 \mathrm{~mA}$ and $z=0$. 
Similar calculations were carried out for a "magnetic capacitor" with the same dimensions at different distances $a$ between the electrodes. It was found that inside the capacitor, decreasing $a$ from $1 \mathrm{~mm}$ to $0.1 \mathrm{~mm}$, the amplitude of the field $h$ and the field inhomogeneity along the longitudinal $y$-axis practically remain unchanged, while the field inhomogeneity in the transverse direction along the $x$-axis decreases significantly. Outside the capacitor, as $a$ decreases from $1 \mathrm{~mm}$ to $0.1 \mathrm{~mm}$, the field is smaller outside the capacitor. In the longitudinal direction near the injection point at $a=0.1 \mathrm{~mm}$, the field decreases by one order of magnitude at a distance of $\sim 0.2 \mathrm{~cm}$ from the electrode edge, and in the transverse direction, at a distance of $\sim 0.3 \mathrm{~cm}$ from the electrode edge.

Summing up, we can conclude that at $a<<b, c$ the alternating field goes beyond the magnetic capacitor by $\sim 2-3$ distances between the electrodes of the capacitor.

With an increase in the frequency of the excitation current, the skin effect can lead to a spatial inhomogeneity of the distribution of the current, and, consequently, the excitation magnetic field in the system. Figures 6 and 7 show the calculated spatial distributions of the current density $j$ of different frequencies over the width and thickness of a copper electrode with a width of $c=10 \mathrm{~mm}$ and a thickness of $50 \mu \mathrm{m}$ in the middle of the excitation system $(x=0)$ at $I=100 \mathrm{~mA}$. From the data shown in Figure 6, it follows that for current frequencies $f$ lower than $\sim 1 \mathrm{MHz}$, the distribution of the current density over the width of the electrode is practically uniform and coincides with the distribution of the direct current density. With an increase in the frequency from $1 \mathrm{MHz}$ to $100 \mathrm{MHz}$, the current density in the central part of the electrode decreases by a factor of $\sim 4$, maintaining a uniform distribution, and near the edges of the electrode in a region $\sim 50 \mu \mathrm{m}$ wide, approximately equal to the thickness of the electrode, the current density increases sharply.

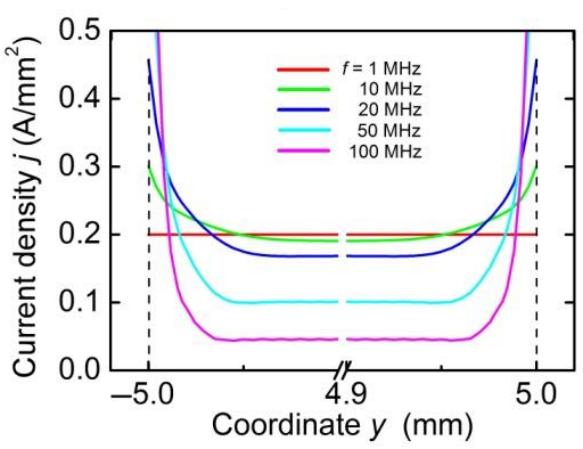

Figure 6. Current density distribution over the width of the $\mathrm{Cu}$ electrode for different current frequencies $f$ from $1 \mathrm{MHz}$ to $100 \mathrm{MHz}$.

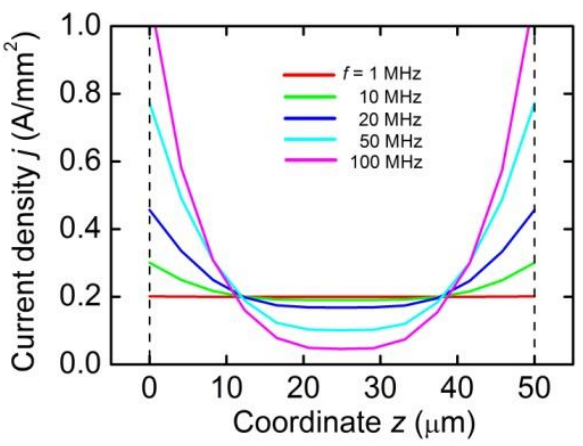

Figure 7. Current density distribution over the thickness of the $\mathrm{Cu}$ electrode for different current frequencies $f$ from $1 \mathrm{MHz}$ to $100 \mathrm{MHz}$.

The redistribution of the current density leads to an increase in the amplitude of the excitation field $h_{\mathrm{y}}$ in a narrow region near the edges of the electrode, and, accordingly, to a decrease in the field in homogeneity over the width of the magnetic capacitor. The distribution of the current density over the thickness of the electrode (Figure 7) is also 
practically uniform at frequencies less than $1 \mathrm{MHz}$. As the frequency increases to $50 \mathrm{MHz}$, the current density decreases in the center of the electrode and increases on the surfaces of the electrode. However, this does not affect the distribution of the magnetic field $h$ in the plane of the excitation system.

It should be added that although the limited dimensions of the magnetic capacitor inevitably lead to inhomogeneity of the excitation field $h_{\mathrm{y}}(x, y)$ between its electrodes, this inhomogeneity is not decisive. The inhomogeneity of the field arising from the effects of demagnetization inside the FM layer [15] can be significantly greater than the inhomogeneity of the field due to the limited size of the magnetic capacitor. Thus, the simulation results show that the magnetic capacitor can operate, in contrast to the coils, at frequencies of tens to hundreds of MHz.

\section{Magnetoelectric Effects in Planar Heterostructures}

To test the applicability of the "magnetic capacitor" system for excitation of the ME effect, several heterostructures with FM and PE layers of various configurations were fabricated and their characteristics were investigated. At the first stage, the efficient excitation of the ME effect in the Metglas/PZT structure by a conducting strip with an alternating current, located near the surface of the structure was demonstrated. Then, the ME effect was investigated in the Metglas/PZT/Metglas heterostructure, which is freely located inside the magnetic capacitor. After that, the $\mathrm{ME}$ effects in monolithic heterostructures of the composition $\mathrm{Cu}$-Metglas/PZT/Metglas- $\mathrm{Cu}, \mathrm{Cu} /$ Metglas-PZT-Metglas/Cu, and $\mathrm{Cu} / \mathrm{NZFO} / \mathrm{Cu}-\mathrm{PZT}$ were investigated. Here, the "/" sign denotes the mechanical and electrical contact between the layers of the structure, and the "-" sign denotes only the mechanical contact between the layers through the dielectric layer. The role of the dielectric was usually played by a layer of glue. At the final stage, the features of the excitation of nonlinear ME effects in a planar Metglas-PZT heterostructure by an alternating current flowing directly through the FM layer were investigated.

\subsection{Materials and Experimental Set-Up}

For experimental studies, several heterostructures of different designs with layers of different materials were made. FM layers of the structures were made of commercially available materials: an amorphous ferromagnet with the composition FeBSiC (Metglas 2605SA1) [16] manufactured by Metglas Co, Germany, and nickel-zinc ferrite with the composition $\mathrm{Ni}_{0.9} \mathrm{Zn}_{0.1} \mathrm{Fe}_{2} \mathrm{O}_{4}$ (NZFO) [17]. The choice of materials is due to the following reasons. The amorphous Metglas alloy is available in the form of ribbons with a thickness of 20-30 $\mu \mathrm{m}$, has a rather high saturation magnetostriction $\lambda_{\mathrm{S}} \approx 25 \times 10^{-6}$ in a low field $\mathrm{H}_{\mathrm{S}}$ $\approx 100 \mathrm{Oe}$, and is a conductor with a resistivity $\gamma_{\mathrm{Mgl}} \approx 1.3 \times 10^{-6} \Omega \cdot \mathrm{m}$. Ferrite NZFO is well processed, and has a saturation magnetostriction $\lambda_{\mathrm{S}}=-24 \times 10^{-6}$ in a saturation field $\mathrm{H}_{\mathrm{S}}$ $\sim 500$ Oe. The high resistivity of the NZFO ferrite $\gamma_{\mathrm{fer}} \approx 10^{8} \Omega \cdot \mathrm{m}$ ensures low losses at high frequencies. Both ferromagnets retain their properties up to temperatures of about $400{ }^{\circ} \mathrm{C}$, making them suitable for fabrication via different technologies. The piezoelectric layers of the structures were made of lead zirconate titanate piezoceramics of the composition $\mathrm{Pb}(\mathrm{Zr}, \mathrm{Ti}) \mathrm{O}_{3}$ (PZT) [18]. The piezoceramic has a piezomodule $\mathrm{d}_{31}=-175 \mathrm{pC} / \mathrm{N}$ and a dielectric constant $\varepsilon \approx 1750$. Ag electrodes, $\sim 3 \mu \mathrm{m}$ thick, were fired on the surface of the PZT layers, and then it was poled along the normal to the plane, applying a voltage of $1 \mathrm{kV}$ for one hour at a temperature of $100{ }^{\circ} \mathrm{C}$. The layers of heterostructures were mechanically connected using cyanoacrylate glue under pressure.

The characteristics of ME effects in heterostructures with coil-free excitation were studied by the magnetic field modulation method [19] using a set-up in which the block diagram is shown in Figure 8. The structure was placed between the poles of an EM electromagnet with a pole diameter of $50 \mathrm{~mm}$ in a permanent magnetic field $H=0-2 \mathrm{kOe}$, which was powered by a GENH600-1.3 current source. An Agilent 33210 generator was used as a source of excitation current I $\cos (2 \pi \mathrm{ft})$ with an amplitude of up to $I=100 \mathrm{~mA}$ and a frequency of $f=1-10 \mathrm{kHz}$. The field $\mathrm{H}$ was measured with a LakeShore 421 gaussmeter with 
an accuracy of 0.1 Oe. The voltage $u$ generated due to the ME effect between the electrodes of the PZT layer of the structure was amplified using an SR850 DSP lock-in amplifier, and measured using an AKIP 2401 voltmeter with an input impedance of $10 \mathrm{M} \Omega$. The frequency spectrum of the generated voltage was measured using an SR760 FFT spectrum analyzer. The dependences of the ME voltage on the frequency $f$ and the amplitude I of the excitation current and the dc bias field $H$ were measured. When studying nonlinear ME effects, the structure was excited with a current of a frequency $f$, and the voltage u was recorded at harmonic frequencies. All measurements were carried out at room temperature. The installation worked in automatic mode under the control of a specialized LabView program. The dependences of the magnetostriction of the FM layers of the structures on the magnetic field $\lambda(H)$ were measured by the tensometric method with an accuracy of $\delta \lambda$ $\approx 0.5 \times 10^{-6}[20]$.

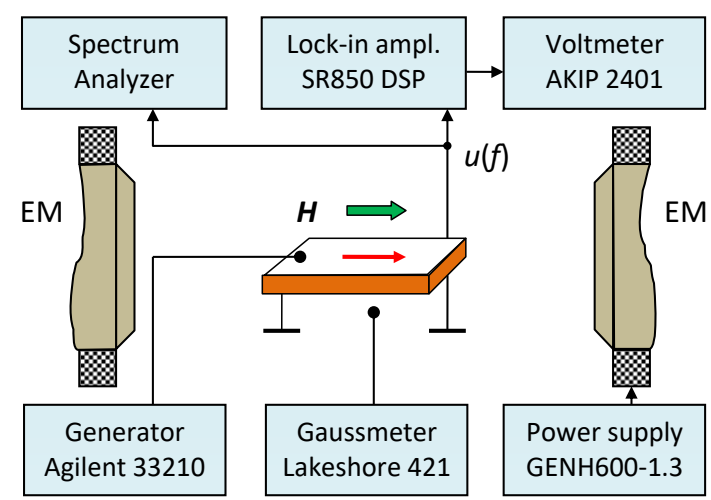

Figure 8. The block diagram of the experimental set-up for the ME effect investigation under coil-free excitation.

\subsection{Excitation of ME Effect by a Strip with Ac Current}

The simplest scheme for the excitation of the ME effect in an FM/PE heterostructure by an alternating current flowing through a strip located near its surface is shown in Figure 9 [21]. The structure used contains a $33 \mathrm{~mm} \times 9 \mathrm{~mm}$ size PZT plate of $\mathrm{a}_{\mathrm{p}}=0.3 \mathrm{~mm}$ thickness with Ag electrodes and a Metglas layer of $33 \mathrm{~mm} \times 5 \mathrm{~mm}$ size and $20 \mu \mathrm{m}$ thick. The Metglas layer and the PZT layer were bonded with cyanoacrylate glue. A glue layer $3 \mu \mathrm{m}$ thick ensured the transfer of deformations from the FM layer to the PZT plate. The structure rested freely on a $\mathrm{Cu}$ strip $9 \mathrm{~mm}$ wide, $50 \mathrm{~mm}$ long, and $50 \mu \mathrm{m}$ thick, which was made by photolithography on an alumina substrate. To provide galvanic isolation between the FM layer of the structure and the Cu strip, a Teflon film $10 \mu \mathrm{m}$ thick was used. An alternating current Icos $(2 \pi \mathrm{ft})$ with an amplitude of up to $I=200 \mathrm{~mA}$ and a frequency of $f=1-100 \mathrm{kHz}$ flows through the strips. The current creates an ac field $h_{y}$ directed along the $y$-axis near the strip surface at the location of the FM layer and excites the ME effect. The voltage $u$ generated due to the ME effect was measured across the Ag electrodes of the PZT layer. A dc field $H=0-100$ Oe was directed parallel to the current along the $x$-axis.

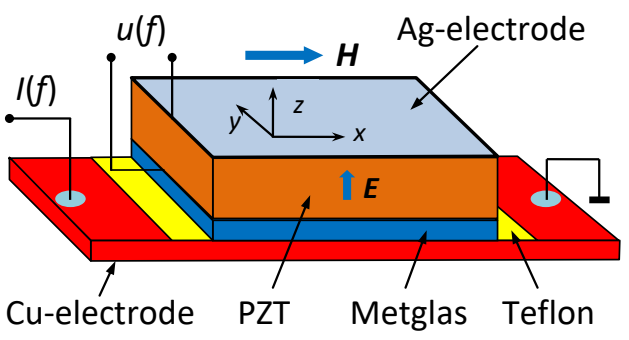

Figure 9. Schematic view of the Metglas/PZT heterostructure excited by a strip with ac current $I$. The arrows show directions of electric $E$ and magnetic $H$ fields. 
Figure 10 shows the dependence of the generated ME voltage $u$ on the current frequency $f$ at $I=200 \mathrm{~mA}$ and a dc bias field $H=5$ Oe. The peak near the frequency $f_{1} \approx$ $46 \mathrm{kHz}$ with magnitude $u_{1} \approx 12 \mathrm{mV}$ and a $Q$-factor of $\sim 90$ at a level of 0.7 corresponds to the excitation of the fundamental mode of longitudinal acoustic vibrations in the structure.

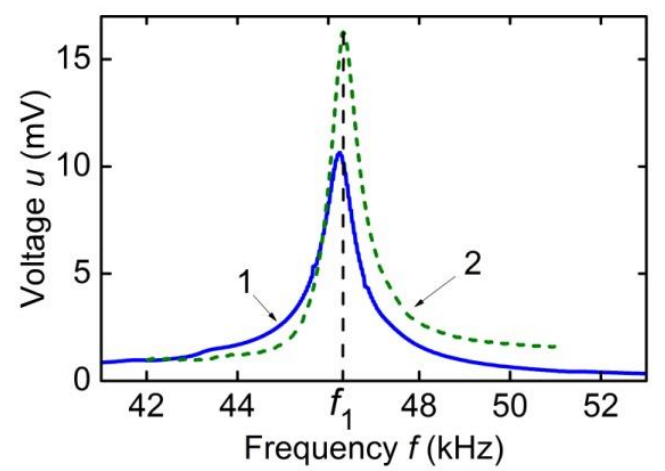

Figure 10. Dependence of ME voltage $u$ on the frequency $f$ upon excitation of a Metglas/PZT structure by a strip with a current (1) and by a volumetric coil (2) at $H=5$ Oe.

Figure 11 shows the dependence of the peak magnitude $u_{1}$ on the bias field $H$. The peak magnitude initially increases with increasing $\mathrm{H}$, reaches a maximum at a characteristic field $H_{m}=5 \mathrm{Oe}$, and then tends to zero as the FM layer becomes saturated. For comparison, Figures 10 and 11 also show the dependence of the ME voltage on the frequency $u(f)$ and the dependence of the resonance peak magnitude on the dc field $u_{1}(H)$ when the heterostructure is excited by a spatially uniform field with an amplitude of $0.25 \mathrm{Oe}$, created by a volumetric coil.

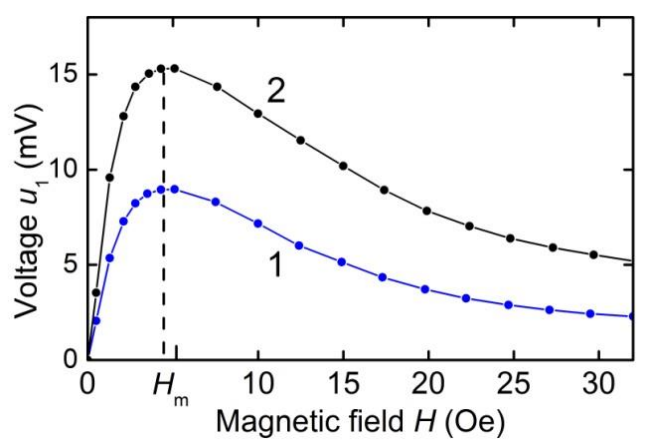

Figure 11. Dependence of ME voltage $u_{1}$ on the field $H$ under the structure excitation at resonance frequency by a strip with a current (1) and a volumetric coil (2).

It can be seen that the characteristics of the ME effect in the described structure upon excitation by a strip with a current qualitatively repeated well the characteristics of the ME effect upon excitation by a volumetric coil.

In both cases, the frequency of the resonance peak was the same, which can be estimated by the formula for the frequency of the fundamental vibration mode of a free plate [22]

$$
f_{1}=(1 / 2 a) \sqrt{Y / b}
$$

Here, $b$ is the length of the structure, and the effective Young's modulus $Y$ and density $\rho$ are found by $Y=\left(Y_{m} a_{m}+Y_{p} a_{p}\right) /\left(a_{m}+a_{p}\right)$ and $\rho=\left(\rho_{m} a_{m}+\rho_{p} a_{p}\right) /\left(a_{m}+a_{p}\right)$, where $a_{m}$ and $a_{p}, Y_{m}$ and $Y_{p}, \rho_{m}$ and $\rho_{p}$ are the thicknesses, Young's moduli, and densities of FM and PE layers, respectively. We neglected the contribution of the electrodes and the adhesive layer because their thicknesses are negligible in comparison with the thicknesses of the layers of the structure. Using the material parameters corresponding to the layers of the structure (Metglas: $Y_{m} \approx 18.6 \times 10^{10} \mathrm{~N} / \mathrm{m}^{2}$ and $\rho_{m} \approx 8.2 \times 10^{3} \mathrm{~kg} / \mathrm{m}^{3}$; PZT: $Y_{p} \approx 7.0$ 
$\times 10^{10} \mathrm{~N} / \mathrm{m}^{2}$ and $\rho_{p} \approx 7.7 \cdot 10^{3} \mathrm{~kg} / \mathrm{m}^{3}$ ), we obtain the frequency resonance $f_{1} \approx 42 \mathrm{kHz}$, which is in good agreement with the measured value.

The amplitude of the voltage generated by the composite FM-PE heterostructure under the direct linear ME effect is qualitatively well described by the formula [23,24]

$$
u(f, H, h) \approx Q d_{31} \lambda^{(1)}(H) h
$$

where $Q$ is the acoustic figure of merit of the structure, $d_{31}$ is the piezomodule of the PE layer, $\lambda^{(1)}(H)=\partial \lambda /\left.\partial H\right|_{H}$ is the piezomagnetic coefficient of the FM layer, $\lambda(H)$ is the dependence of the magnetostriction of the FM layer on the magnetic field $H, \mathrm{~h}$ is the amplitude of the excitation magnetic field.

From (2), it follows that the shape of the field dependence of the ME voltage $u(H)$ (see Figure 11) is determined by the field dependence of the piezomagnetic coefficient of the FM layer of the structure. The piezomagnetic coefficient increases with increasing $H$, reaches its maximum value at a certain field $H_{m} \approx 4.5 \mathrm{Oe}$, and then tends to zero as the FM layer of the structure becomes saturated. Estimation of the $\mathrm{ME}$ coefficient at the resonance frequency gives $\alpha_{E}=u /\left(h \cdot a_{p}\right) \approx 1 \mathrm{~V} /($ Oe $\cdot \mathrm{cm})(h \approx 0.13$ Oe at $I=200 \mathrm{~mA})$ when the structure is excited by a current through the strips and $1.5 \mathrm{~V} /(\mathrm{Oe} \cdot \mathrm{cm})$ when the structure is excited with a volumetric coil.

Thus, one strip with an ac current, located near the FM-PE heterostructure, rather effectively excites the ME effect in it. The disadvantage of the excitation of the ME effect by the strip with ac current is that the magnetic field from the strip decreases slowly $h$ $\sim 1 / \mathrm{r}$ with increasing distance $\mathrm{r}$ to the strip, therefore it creates a large electromagnetic interference in the surrounding space.

\subsection{ME Effect in a Metglas/PZT/Metglas Heterostructure Placed in a Magnetic Capacitor}

The design of the Metglas/PZT/Metglas heterostructure with an excitation system in the form of a "magnetic capacitor" is shown schematically in Figure 12 [25]. The structure contains a square PZT plate with an in-plane dimension $b=15 \mathrm{~mm}$ and a thickness $a_{p}=1 \mathrm{~mm}$. On both surfaces of the PZT plate, layers of Metglas are glued with an in-plane size of $15 \mathrm{~mm} \times 15 \mathrm{~mm}$ and a thickness of $a_{m}=23 \mu \mathrm{m}$. The structure is placed inside a magnetic capacitor with $16 \mathrm{~mm} \times 16 \mathrm{~mm}$ planes made of $\mathrm{Cu}$ foil, $30 \mu \mathrm{m}$ thick. The distance between the $\mathrm{Cu}$ planes was $a=1050 \mu \mathrm{m}$. An ac current Icos $(2 \pi \mathrm{ft})$ with an amplitude of up to $I=100 \mathrm{~mA}$, flowing along the $x$-axis, is supplied to the contacts of the magnetic capacitor. The current generates between the plates of the capacitor an alternating field hos $(2 \pi \mathrm{ft})$ directed along the $y$-axis. In fact, instead of a volumetric multi-turn coil, one turn of a strip with a current is used. The heterostructure is located freely inside the magnetic capacitor and is isolated from the electrodes by a Teflon tape $10 \mu \mathrm{m}$ thick. This ensured effective excitation of planar acoustic vibrations of the structure and galvanic isolation of the PZT plate from the $\mathrm{Cu}$ electrode. The excitation system had an active resistance of $\mathrm{R}_{1} \approx 30 \mathrm{~m} \Omega$ and an inductance $\mathrm{L}=21 \mu \mathrm{H}$, measured at a frequency of $10 \mathrm{kHz}$. A dc field $\mathrm{H}$ is applied along the $y$-axis.

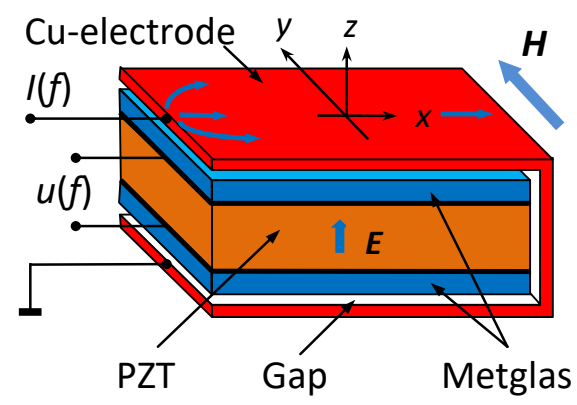

Figure 12. Schematic view of a Metglas/PZT/Metglas heterostructure placed inside the "magnetic capacitor". The arrows show directions of electric $E$ and magnetic $H$ fields. 
Figure 13 shows the distributions of the $h_{y}$ field component along the length and width of the magnetic capacitor. The distributions were recorded using a probe coil with a cross-sectional area of $1 \mathrm{~mm}^{2}$ and a number of turns, 50, at a current amplitude of $I \approx$ $80 \mathrm{~mA}$ and a frequency of $132 \mathrm{kHz}$. It can be seen that the field amplitude in the center between the planes is $h_{y} \approx 60 \mathrm{mOe}$, and decreases somewhat when approaching the edges of the plates, which qualitatively agrees with the theoretical calculations (see Figure 5). An estimate of the field amplitude gives a value of $60 \mathrm{mOe}$, which is in good agreement with the measured one. When approaching the edges of the magnetic capacitor, the field amplitude drops sharply in accordance with the calculation. The measured amplitude of the magnetic field outside the electrodes of the magnetic capacitor near their surfaces did not exceed $0.1 \mathrm{mOe}$.

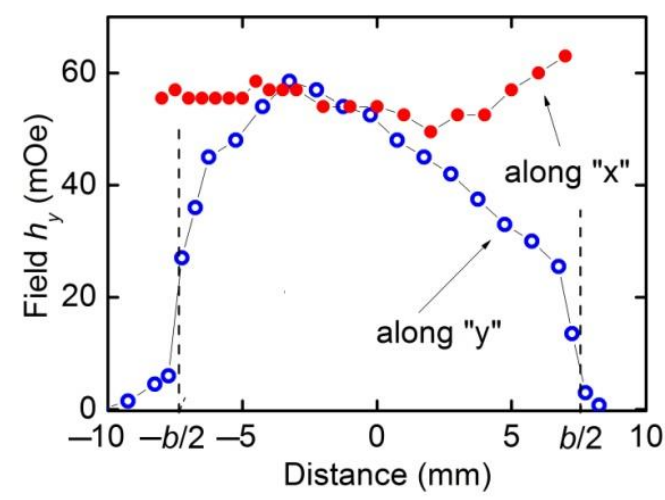

Figure 13. Measured distributions of excitation field $h_{\mathrm{y}}$ along and across the direction of the current in a "magnetic capacitor" for $I=80 \mathrm{~mA}$. Curve along " $x$ " is for $y=0$ and $z=0$, and curve along " $y$ " is for $x=0$ and $z=0$.

Figure 14 shows the dependence of the ME voltage $\mathrm{u}$ on the current frequency $\mathrm{f}$ at the current amplitude $I=80 \mathrm{~mA}$ and the optimal bias field $H_{m}=30$ Oe. The voltage peak with a central frequency $f_{1} \approx 132.7 \mathrm{kHz}$, magnitude $u_{1}=91 \mathrm{mV}$, and quality factor $Q_{1} \approx 10^{2}$ corresponds to the excitation of the fundamental mode of planar oscillations of the structure. The estimation of the resonance frequency at the values of the structure parameters corresponding to the experiment gives the frequency $130 \mathrm{kHz}$, which is in good agreement with the measured one. The ME conversion coefficient at the resonance frequency was $10 \mathrm{~V} /(\mathrm{Oe} \cdot \mathrm{cm})$, which is typical for PZT/Metglas heterostructures upon excitation with volumetric coils [26].

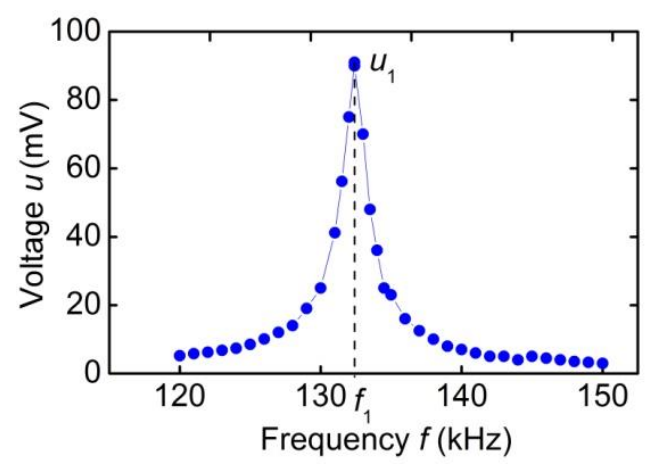

Figure 14. Dependence of ME voltage $u$ on the frequency $f$ of the excitation current for the Metglas/PZT/Metglas heterostructure, placed in a "magnetic capacitor".

Figure 15 shows the dependence of the ME voltage $u_{1}$ on the bias field $H$ at the resonance frequency for the amplitude of the excitation current $I=80 \mathrm{~mA}$. The maximum conversion was observed in a field $H_{m} \approx 30 \mathrm{Oe}$, corresponding to the maximum of the piezomagnetic coefficient of the Metglas layer. The magnitude of the field $H_{m}$ is largely 
determined by the magnitude of the saturation magnetization and the demagnetization effects of the FM layers and can be reduced to 2-10 Oe, by increasing the size or decreasing the thickness of the FM layers.

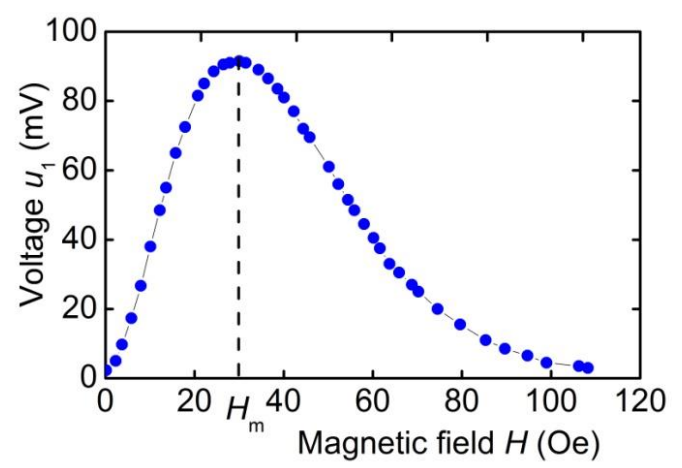

Figure 15. Dependence of ME voltage $u$ on the magnetic field $H$ for the Metglas/PZT/Metglas heterostructure placed in a "magnetic capacitor".

Thus, the data presented indicate that the resonant ME effect in the composite heterostructure is effectively excited by the "magnetic capacitor" type system.

\subsection{ME Effect in a Monolithic Cu-Metglas/PZT/Metglas-Cu Heterostructure}

Now let us consider a monolithic Cu-Metglas/PZT/Metglas-Cu heterostructure in which, in contrast to the construction described in the previous section, the "magnetic capacitor" excitation system is integrated into the heterostructure, as shown in Figure 16 [14]. The monolithic heterostructure contains a PZT plate with a length $b=21 \mathrm{~mm}$, width $c=4 \mathrm{~mm}$, and a thickness $a_{p}=200 \mu \mathrm{m}$, located between Metglas layers of the same dimensions and a thickness $a_{m}=25 \mu \mathrm{m}$. The PZT plate and the Metglas layers are glued together. The whole structure is coated with a thin layer of photocurable Mechanic LYUVH900 polymer and placed inside a magnetic capacitor with $\mathrm{Cu}$ electrodes, $20 \mathrm{~mm} \times$ $4 \mathrm{~mm}$ in size and $20 \mu \mathrm{m}$ thick. The structure and electrodes of the capacitor are connected under pressure with glue. Glue layers, $\sim 3 \mu \mathrm{m}$ thick, ensured mechanical contact between all layers of the structure, and the polymer played the role of a dielectric and provided electrical isolation between the Metglas layers and $\mathrm{Cu}$ electrodes. An electric current Icos ( $2 \pi \mathrm{ft}$ ) with an amplitude of up to $I=100 \mathrm{~mA}$ and a frequency of $f=1 \mathrm{kHz}-1 \mathrm{MHz}$ was supplied through contacts at the left end of the electrodes. The active resistance of the magnetic capacitor was $R \approx 0.1 \Omega$. The current creates in the FM layers an excitation field $h$ with an amplitude of up to 0.4 Oe, directed perpendicular to the long axis of the structure. A dc field $H=0-1 \mathrm{kOe}$ is applied tangentially to the plane of the structure along its length or width.

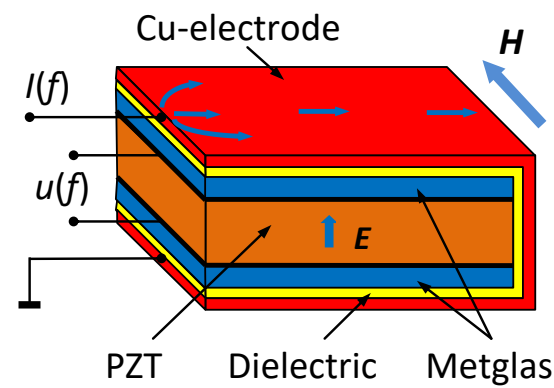

Figure 16. Schematic view of a $\mathrm{Cu}$ /Metglas-PZT-Metglas/Cu heterostructure with integrated "magnetic capacitor" excitation system. The arrows show directions of electric $E$ and magnetic $H$ fields.

Figure 17 shows the characteristics of a monolithic heterostructure upon magnetization by a field $\mathrm{H}$ across the long axis, i.e., perpendicular to the direction of the current $H \perp I$. 
Figure 17a shows the dependence of the ME voltage amplitude $u$ on the current frequency f at $I=100 \mathrm{~mA}$ and $H=134$ Oe. The peak with a frequency of $f_{1}=74 \mathrm{kHz}$, a magnitude of $u_{1}=120 \mathrm{mV}$, and a quality factor of $Q_{1}=96$ corresponds to the excitation of the fundamental mode of planar acoustic vibrations along the length of the structure. The peak with a frequency of $f_{2} \approx 438 \mathrm{kHz}$, a magnitude of $u_{2}=120 \mathrm{mV}$, and quality factor of $Q_{2} \sim 100$ corresponds to the excitation of the fundamental mode of planar acoustic vibrations along the width of the structure. Figure $17 \mathrm{~b}$ shows the dependence of the magnitude of the low-frequency peak $\mathrm{u}_{1}$ on the field $\mathrm{H}$ at a current $I=100 \mathrm{~mA}$. It can be seen that the voltage $\mathrm{u}_{1}$ initially increases with increasing $\mathrm{H}$, reaches a maximum at a characteristic field of $H_{m}^{\perp} \approx 134$ Oe, and then monotonically tends to zero as the FM layer is saturated. The field $H_{m}^{\perp}$ corresponds to the maximum of the piezomagnetic coefficient of the Metglas layer of the structure. The magnitude of the ME coefficient in this case, according to estimates, was $\alpha_{E} \approx 8.6 \mathrm{~V} /(\mathrm{Oe} \cdot \mathrm{cm})$ at both frequencies.
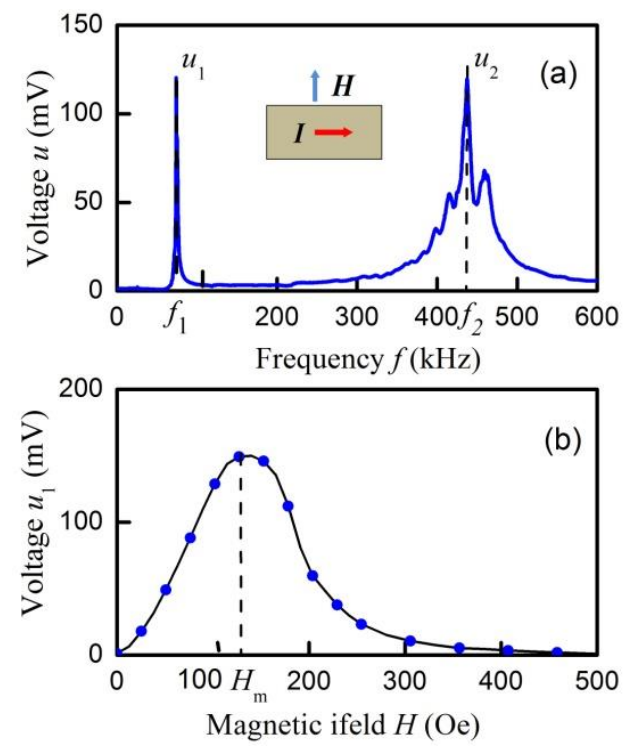

Figure 17. Characteristics of a monolithic Cu-Metglas/PZT/Metglas-Cu structure with integrated excitation system at $H \perp I$; (a) ME voltage $u$ vs. frequency $f$ for $H=134$ Oe; (b) ME voltage $u_{1}$ vs. field $H$ for $f=73.3 \mathrm{kHz}$.

Figure 18 shows the characteristics of the heterostructure upon magnetization by the field $\mathrm{H}$ along the long axis, i.e., along the direction of the current, $H /$ / I. From Figure 18a, it can be seen that $u(f)$ dependence contains two peaks with the same frequencies. The peak of a frequency $f_{1}$ has a magnitude of $u_{1}=28 \mathrm{mV}$ and a quality factor of $Q_{1} \approx 105$, and a peak of a frequency of $f_{2}$ has a magnitude of $u_{2}=5 \mathrm{mV}$ and a quality factor of $Q_{2} \approx 30$. The voltage amplitudes are $\sim 4$ and $\sim 24$ times, respectively, less than when the structure is magnetized across the long axis. The dependence of the magnitude of the peak with frequency $\mathrm{f}_{1}$ on the field $H$ (see Figure 18b) has the same form as in Figure 17b, however, the maximum voltage $u_{1} \approx 28 \mathrm{mV}$ occurs at a lower field of $\sim 18$ Oe. The difference in characteristic fields is because the magnitude of the demagnetizing field when the structure is magnetized along the long axis is much less than when the structure is magnetized in the transverse direction [27]. The voltage amplitude at the resonance frequency linearly depended on the excitation current, with a coefficient $u / I=0.25 \mathrm{~V} / \mathrm{A}$. The magnitude of the $\mathrm{ME}$ coefficient at resonant frequency $\mathrm{f}_{1}$ was $\alpha_{E} \approx 2.2 \mathrm{~V} /($ Oe $\cdot \mathrm{cm})$. 

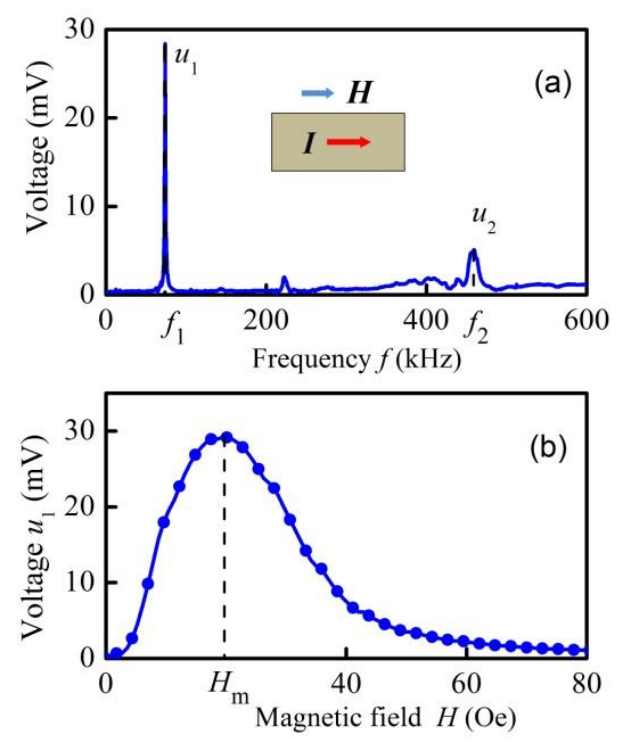

Figure 18. Characteristics of a monolithic Cu-Metglas/PZT/Metglas-Cu structure with integrated excitation system at $H / / I$; (a) ME voltage $u$ vs. frequency $f$ for $H=20$ Oe; (b) ME voltage $u$ vs. field $H$ for $f=73.3 \mathrm{kHz}$.

The data presented indicate that the "magnetic capacitor" excitation system provides high efficiency of the ME effect excitation when it is magnetized perpendicular to the direction of the current, i.e., when the excitation field is $h / / H$. In this case, due to the low inductance of the "magnetic capacitor", the efficiency of the ME effect excitation does not decrease with increasing frequency (see Figure 17a). It follows that a magnetic capacitor can be used to excite ME effect in heterostructures at higher frequencies than magnetic coils allow.

\subsection{ME Effect in a Monolithic Cu/Metglas-PZT-Metglas/Cu Heterostructure}

Another design of a monolithic $\mathrm{Cu} /$ Metglas-PZT-Metglas/Cu heterostructure, in which the "magnetic capacitor" excitation system is also integrated into the structure, is shown schematically in Figure 19. It contains a square-shaped PZT plate with side $b=15 \mathrm{~mm}$ and thickness $a_{p}=0.5 \mathrm{~mm}$, located between two Metglas layers. Metglas layers have the same in-plane dimensions and thickness $a_{m}=25 \mu \mathrm{m}$. Cu electrodes with a thickness of $\sim 2 \mu \mathrm{m}$ are deposited on the outer surfaces of the Metglas layers by the electrolytic method. All layers of the structure are connected under pressure using cyanoacrylate glue. The adhesive layer, located between the Metglas layers and the electrodes of the PZT plate, transmits deformations and at the same time acts as a dielectric, providing galvanic isolation between the excitation system and the PZT plate. A dc field $\mathrm{H}$ is applied in the plane of the structure perpendicular to the current I. On the left side, an alternating current with an amplitude of up to I $=100 \mathrm{~mA}$ is supplied to the Cu electrodes, and the right ends of the electrodes are connected to each other. Since the resistivity of copper $\gamma_{\mathrm{Cu}}=1.7 \times 10^{-8} \Omega \cdot \mathrm{m}$ is much less than the resistivity of Metglas $\gamma_{\mathrm{Mgl}}=1.3 \times 10^{-6}$ $\Omega \cdot \mathrm{m}[18]$, the current flows mainly through the $\mathrm{Cu}$ electrode and creates an excitation field $h / / H$. 


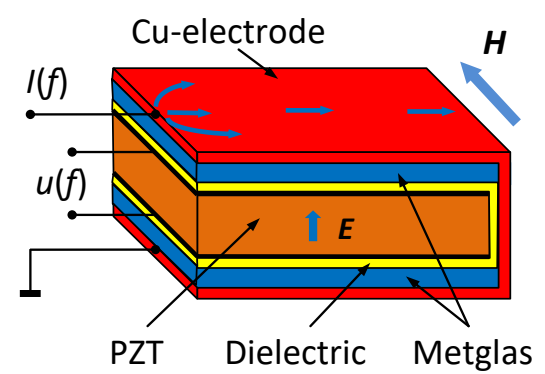

Figure 19. Schematic view of a monolithic $\mathrm{Cu}$ /Metglas-PZT-Metglas/ $\mathrm{Cu}$ heterostructure with an integrated "magnetic capacitor" excitation system. The arrows show directions of electric $E$ and magnetic $H$ fields.

Figure 20 shows the dependence of the ME voltage $u$ on the frequency $f$ of the excitation current for $I=100 \mathrm{~mA}$ and the bias field $H=25$ Oe. The voltage peak near the frequency $f_{1}=124 \mathrm{kHz}$ with a magnitude of $u_{1}=200 \mathrm{mV}$ and a quality factor of $Q_{1} \approx 150$ corresponds to the excitation of the fundamental mode of planar acoustic vibrations in the structure. Estimation of the resonance frequency at the corresponding experimental values of the parameters gives a frequency of $\sim 130 \mathrm{kHz}$, which is in good agreement with the measured one. The irregularity of the line may be due to the difference in the shape of the structure from the square. Figure 21 shows the dependence of the voltage peak magnitude $\mathrm{u}_{1}$ on the magnetic field $\mathrm{H}$ for the excitation current $I=100 \mathrm{~mA}$. The field dependence has a form typical for ME effect, which is determined by the form of the field dependence of the piezomagnetic coefficient. The field $H_{m} \approx 25$ Oe corresponds to the field of the maximum of the piezomagnetic coefficient. The voltage amplitude at the resonance frequency linearly depended on the exciting current with a coefficient $u / I=2 \mathrm{~V} / \mathrm{A}$. The magnitude of the ME coefficient at frequency $\mathrm{f}_{1}$ was $\alpha_{E} \approx 24 \mathrm{~V} /(\mathrm{Oe} \mathrm{cm})$.

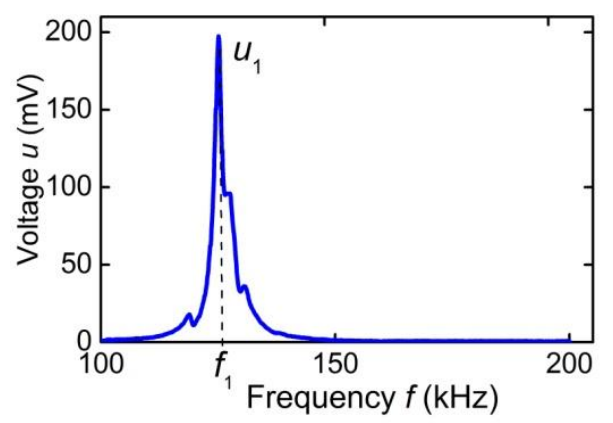

Figure 20. Dependence of the ME voltage $u$ on the excitation current frequency $f$ for the $\mathrm{Cu} / \mathrm{Metglas}-$ PZT-Metglas $/ \mathrm{Cu}$ heterostructure at $H=25$ Oe.

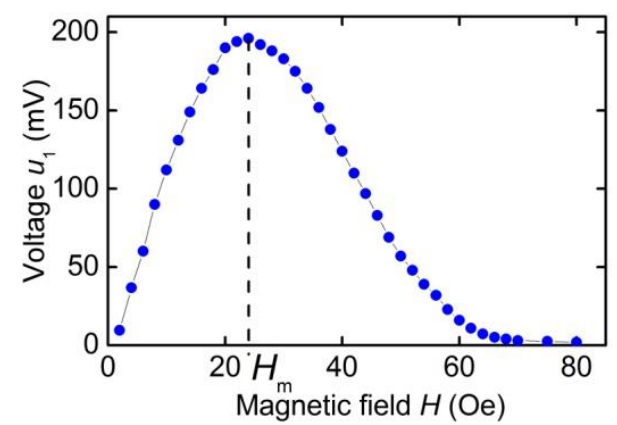

Figure 21. Dependence of the ME voltage $u_{1}$ on the field $H$ for the $\mathrm{Cu} /$ Metglas-PZT-Metglas/Cu heterostructure at frequency $f_{1}$. 
The above results indicate that the described design of a monolithic planar structure with an integrated excitation system of the "magnetic capacitor" type provides effective excitation of the ME effect.

\subsection{ME Effect in a Monolithic Cu/NZFO/Cu-PZT Heterostructure}

Finally, Figure 22 shows the design of a monolithic $\mathrm{Cu} / \mathrm{NZFO} / \mathrm{Cu}-\mathrm{PZT}$ heterostructure, in which the excitation system is also integrated [28]. In contrast to the previously described designs, here only the FM layer is located inside the magnetic capacitor. The heterostructure contains a plate of nickel-zinc ferrite NZFO with dimensions in the plane of $14 \mathrm{~mm} \times 15.5 \mathrm{~mm}$ and a thickness of $a_{m}=2.5 \mathrm{~mm}$ and a PZT plate with dimensions of $15.5 \mathrm{~mm} \times 14 \mathrm{~mm}$ and a thickness of $0.5 \mathrm{~mm}$ with Ag electrodes. A Cu electrode, $2 \mu \mathrm{m}$ thick, was deposited on both planes and one side face of the NZFO plate by chemical deposition. The resistance and inductance of the $\mathrm{Cu}$ electrode, measured at a frequency of $1 \mathrm{kHz}$, were $R=0.021 \Omega$ and $L=22 \mu \mathrm{H}$, respectively. The layers of the structure are coupled together using cyanoacrylate glue. An adhesive layer, $\sim 3 \mu \mathrm{m}$ thick, between the $\mathrm{Cu}$ electrode and the Ag electrode of the PZT-plate, transmits deformations and at the same time acts as a dielectric, providing galvanic isolation. A dc bias magnetic field $\mathrm{H}$ is applied perpendicular to the direction of the current.

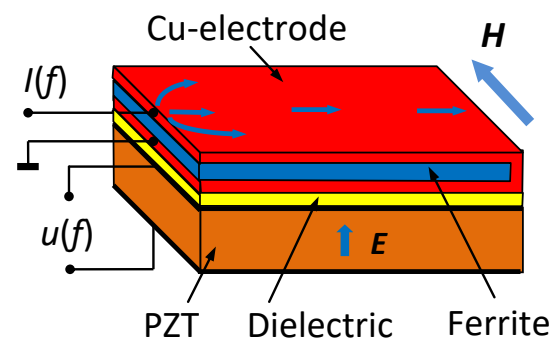

Figure 22. Schematic view of a $\mathrm{Cu} / \mathrm{NZFO} / \mathrm{Cu}-\mathrm{PZT}$ heterostructure with integrated excitation system. The arrows show directions of electric $E$ and magnetic $H$ fields.

Figure 23 shows the dependence of the ME voltage $\mathrm{u}$ generated by the structure on the frequency $\mathrm{f}$ of the excitation current for a bias field $H=95$ Oe and current $I=100 \mathrm{~mA}$. Voltage peak with a magnitude of $u_{1} \approx 170 \mathrm{mV}$, a frequency of $f_{1}=187 \mathrm{kHz}$, and a quality factor of $Q_{1}=120$ corresponds to the excitation of the lowest acoustic modes along the length of the structure. It is seen that the current effectively excites the resonant ME effect at the fundamental mode of acoustic vibrations.

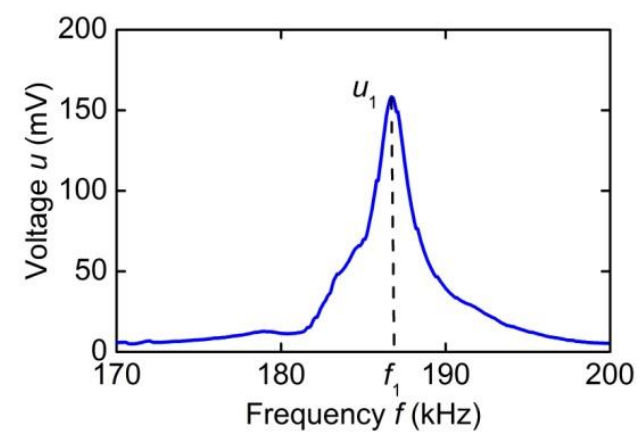

Figure 23. Dependence of ME voltage $u$ on the current frequency $f$ for a monolithic $\mathrm{Cu} / \mathrm{NZFO} / \mathrm{Cu}$ PZT heterostructure with an integrated excitation system at $I=0.1 \mathrm{~A}$ and $H=95$ Oe.

Figure 24 shows the dependence of the ME voltage $\mathrm{u}_{1}$ on the bias field $H$ at $I=100 \mathrm{~mA}$. The dependence has a form characteristic of the linear ME effect: the voltage $\mathrm{u}_{1}$ increases approximately linearly with increasing $\mathrm{H}$, reaches a maximum at a characteristic field $H_{m}$ $\approx 95 \mathrm{Oe}$, and then gradually decreases with the saturation of the FM layer. When the direction of the field $\mathrm{H}$ is inverted, there is a hysteresis in the $\mathrm{ME}$ effect, the magnitude 
of which does not exceed $H_{C} \approx 2$ Oe. The responsivity of the ME effect to the excitation current at a bias field $H_{m}$ is $S_{I}=u_{1} / I \approx 1.7 \mathrm{~V} / \mathrm{A}$. An estimate using Figure 23 for a given ME structure gives a ME coefficient of $\alpha_{E} \approx 42 \mathrm{~V} /(\mathrm{Oe} \cdot \mathrm{cm})$, which is comparable to the ME coefficients for NZFO/PZT structures upon excitation with volumetric coils [13,29]. This indicates that it is promising to use the "magnetic capacitor" excitation system, covering only the magnetic layer, in order to excite the ME effect in planar heterostructures.

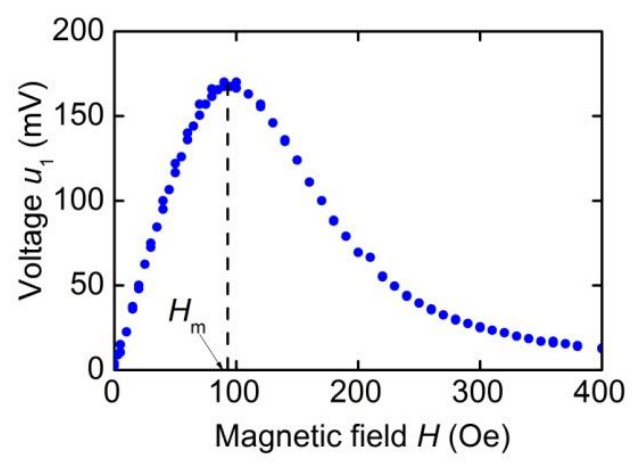

Figure 24. Dependence of ME voltage $u$ on the field $H$ for a monolithic $\mathrm{Cu} / \mathrm{NZFO} / \mathrm{Cu}-\mathrm{PZT}$ heterostructure with integrated excitation system at a frequency $f_{1}$.

Summarizing the results of the studies described in Sections 3.3-3.6, we can conclude that the ME coefficient for heterostructures with an excitation system "magnetic capacitor" is comparable to the ME coefficient of $\sim 1-10^{2} \mathrm{~V} /(\mathrm{Oe} \cdot \mathrm{cm})$ for the structures excited with volumetric coils (see Figure 10 in [30]).

\subsection{Excitation of ME Effect by a Current Flowing through the FM Layer}

In conclusion, let us consider the excitation of nonlinear ME effects in heterostructures, in particular, the generation of harmonics, without using volumetric coils. It was shown in $[31,32]$ that this can be done by passing an alternating current directly through a layer of a conducting ferromagnet.

The measurements were performed using the structure schematically shown in Figure 25 [32]. The structure contains a PZT plate $b=33 \mathrm{~mm}$ long, $c=1.5 \mathrm{~mm}$ wide, and $a_{p}=300 \mu \mathrm{m}$ thick, with Ag electrodes and a Metglas layer $33 \mathrm{~mm}$ long, $c_{m}=1.5 \mathrm{~mm}$ wide, and $a_{m}=20 \mu \mathrm{m}$ thick. The layers of the structure are connected with cyanoacrylate glue. A glue layer $\sim 5 \mu \mathrm{m}$ thick transferred deformations and at the same time played the role of a dielectric, providing isolation of the ferromagnet from the Ag electrode of the PZT plate. An ac current with an amplitude of up to $I=200 \mathrm{~mA}$ and a frequency of $f=0-100 \mathrm{kHz}$ was passed through the ferromagnetic layer. The structure is placed in a dc bias field $\mathrm{H}$ directed along its long side.

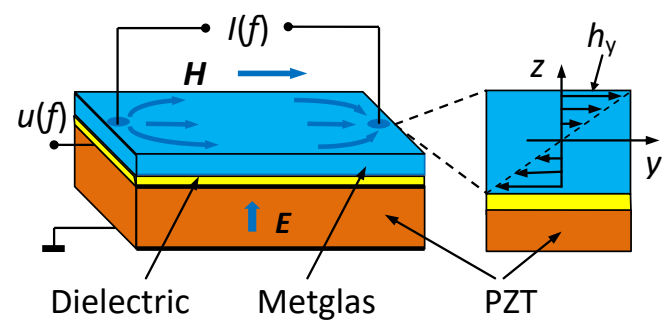

Figure 25. Schematic view of a FeBSiC/PZT heterostructure upon excitation of the ME effect by alternating current flowing through the FM layer. The arrows show directions of electric $E$ and magnetic $H$ fields.

Figure 26 shows the dependence of the ME voltage $u$ on the frequency of the excitation current $\mathrm{f}$ for $I=200 \mathrm{~mA}$ and $H=0.6$ Oe. Three peaks are seen with frequencies $f_{1} \approx$ $46.3 \mathrm{kHz}, f_{2}=f_{1} / 2 \approx 23.0 \mathrm{kHz}$, and $f_{4}=f_{1} / 4 \approx 11.5 \mathrm{kHz}$ of approximately the same quality 
factors $\mathrm{Q} \approx 80$. It was verified that the voltage $\mathrm{u}$ at all three peaks had the same frequency $f_{1} \approx 46.3 \mathrm{kHz}$. An estimate of the frequency of the longitudinal acoustic resonance of the structure gave a value of $47.8 \mathrm{kHz}$, which is in good agreement with $f_{1}$. Hence it follows that the peak at the frequency of the excitation current $f_{1}$ corresponds to the generation of the fundamental harmonic of the ME voltage with the amplitude $u_{1}$, the peak at the frequency of the current $f_{2}$ corresponds to the generation of the second harmonic with the amplitude $u_{2}$, and the peak at the current frequency $f_{4}$ corresponds to the generation of the fourth harmonic with the amplitude $u_{4}$. The structure most efficiently generates the second voltage harmonic, which was also observed in [33].

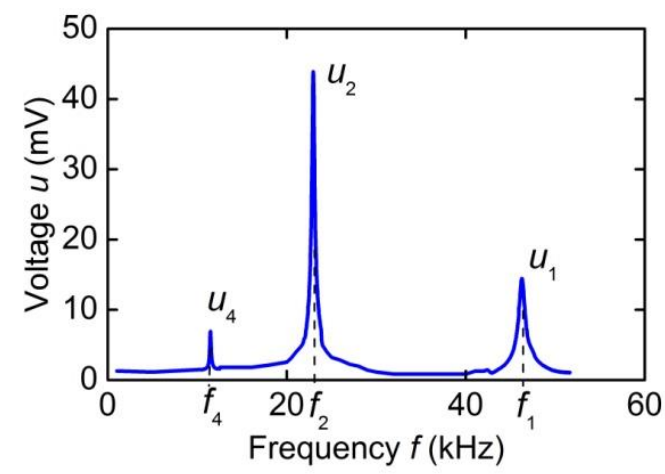

Figure 26. Dependence of ME voltage $u$ on the current frequency $f$ for the Metglas/PZT heterostructure excited by a current flowing through the Metglas layer.

Figure 27 shows the dependences of the amplitudes of the second $u_{2}(\mathrm{H})$ and fourth $u_{4}(\mathrm{H})$ harmonics of the ME voltage on the field $\mathrm{H}$ for a current of $I=200 \mathrm{~mA}$. The second harmonic was excited by a current with a frequency of $23 \mathrm{kHz}$, and the fourth, by a current with a frequency of $11.5 \mathrm{kHz}$. It can be seen that the second harmonic has a maximum amplitude of $u_{2} \approx 42.3 \mathrm{mV}$ in the absence of a field and then decreases monotonically approximately according to the law $u_{2} \sim 1 / H$. The inset in Figure 27 shows that the amplitude of the second harmonic increases quadratically with an increase in the excitation current, $u_{2} \sim I^{2}$.

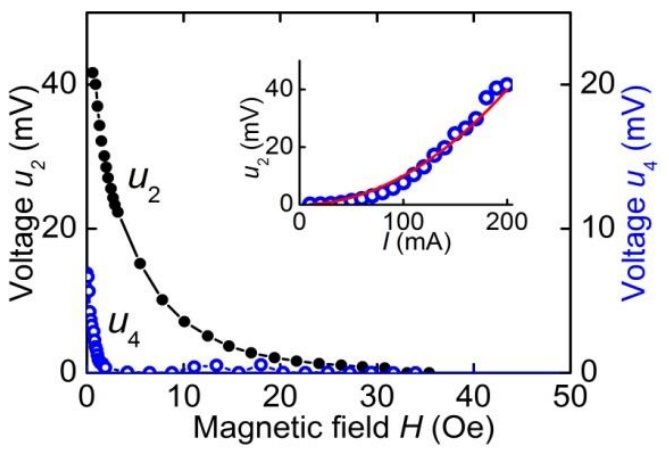

Figure 27. Dependences of ME voltage $u$ on the field $H$ and excitation field $h$ for the Metglas/PZT heterostructure excited by a current flowing through the Metglas layer.

To explain the features of the excitation of the ME effect in the considered heterostructure, we find the field $\mathrm{h}$ created by the current in the FM layer. Using the theorem on the circulation of the magnetic field and assuming the current density $j=I /\left(a_{m} c_{m}\right)$ to be constant, we obtain $h(z)=I z /\left(a_{m} c_{m}\right)$. Thus, the field $h$ is equal to zero at the midline of the FM layer at $z=0$, varies linearly over the layer thickness, and the field directions are opposite on its surfaces at $z= \pm a_{m} / 2$, as shown in Figure 25. At a current $I=200 \mathrm{~mA}$, the excitation field is maximal on the surfaces of the FM layer, $h_{\max } \approx 0.8 \mathrm{Oe}$, and the field amplitude averaged over the layer thickness is 0.4 Oe. This magnetic field leads to magnetostrictive deformation of the FM layer along the width, the deformation has the 
same sign throughout the entire thickness of the FM layer and is transmitted through the interface to the PZT layer.

When the ME effect is excited by a current flowing through the FM layer, in contrast to the excitation of the ME effect by an external field, demagnetization is absent and the field inside the ferromagnet is not weakened [32]. This leads to higher efficiency of the current excitation of the ME effect. Using the data of Figure 26 and the average field $h=0.4$ Oe at $I=200 \mathrm{~mA}$, we find the nonlinear ME coefficient for the excitation of the second voltage harmonic $\alpha_{E}^{(2)}=u_{2} /\left(h^{2} a_{p}\right) \approx 8.2 \mathrm{~V} /\left(\mathrm{cm} \cdot \mathrm{Oe}^{2}\right)$, which is greater than the excitation of the second harmonic by a volumetric coil [31] by a factor of $\sim 17$.

The field dependence of the second harmonic $u_{2}(H)$ upon the excitation of the ME effect in heterostructures by a current through the FM layer is qualitatively different from the analogous dependence upon the excitation of the ME effect with volumetric coils or a "magnetic capacitor" system. This is primarily since the current creates an excitation field hoos $(2 \pi f t)$, which is directed perpendicular to the dc field H. It was shown in [34] that in this case, the structure generates an ME voltage of the form

$$
u(f) \approx A d_{31}\left(\lambda^{(1)} \frac{h^{2}}{4 H}+\lambda^{(2)} \frac{h^{4}}{16 H^{2}}\right) \cos (4 \pi t)+A d_{31} \lambda^{(2)} \frac{h^{4}}{64 H^{2}} \cos (8 \pi t)+\ldots
$$

where $\mathrm{A}$ is the coefficient determined by the shape, size, and dielectric parameters of the structure layers, $d_{31}$ is the piezomodule of the PE layer of the structure, $\lambda^{(1)}(H)=\partial \lambda / \partial H$ is the piezomagnetic coefficient of the FM layer, and $\lambda^{(2)}(H)=\partial^{2} \lambda / \partial H^{2}$ is the nonlinear piezomagnetic coefficient of the FM layer. From (3) it follows that: upon excitation by a current through the magnetic layer, the heterostructure should generate only even voltage harmonics. The amplitude of the second harmonic contains two terms, and the first one is decisive since $\lambda^{(1)}$ is an order of magnitude larger than $\lambda^{(2)}$. The amplitude of the fourth harmonic $u_{4} \sim \lambda^{(2)} h^{4} / H^{2}$ decreases rapidly with increasing $H$.

The theoretically predicted high efficiency of generation of the second harmonic of the ME voltage, the dependence of its amplitude on a dc field of the form $u_{2} \sim 1 / H$, and the quadratic dependence on the excitation field $u_{2} \sim h^{2}$ are in good agreement with the measurement results. The generation of the weak first harmonic of the ME voltage observed experimentally (see Figure 26) can be caused by both the Poisson effect and the non-orthogonality of the fields $\mathrm{h}$ and $\mathrm{H}$, due to the anisotropy of the FM layer.

\section{Conclusions}

The research results demonstrate the possibility of excitation of the ME effect in planar heterostructures with FM and PE layers without using volumetric electromagnetic coils. Instead of coils for generating an alternating magnetic field, an excitation system of the "magnetic capacitor" type can be used. The magnetic capacitor can be integrated into the heterostructure. Simulations and measurements have shown that the magnetic field is concentrated mainly inside the magnetic capacitor, being rather homogeneous in space, and rapidly decreasing with distance outside the edges of the capacitor. Monolithic planar heterostructures of various designs with PE layers of PZT piezoelectric ceramics and FM layers of amorphous alloy Metglas or NZFO ferrite with an integrated excitation system were fabricated and investigated. The ME conversion coefficient for planar structures obtained was $1-40 \mathrm{~V} /(\mathrm{Oe} \cdot \mathrm{cm})$, which is comparable to the ME coefficient for structures of a similar composition upon excitation with volumetric coils. The small volume and low impedance of the proposed excitation system make it possible to increase the operating frequencies of ME devices up to hundreds of $\mathrm{MHz}$. The next step is the development and study of monolithic ME structures with an integrated excitation system containing films of various FM and PE materials with high magnetostriction and high piezoelectric effect [35-37]. The use of planar technologies mastered in semiconductor technology for the manufacture of ME devices will accelerate their implementation in modern electronics and microsystem technology. 
Author Contributions: Conceptualization, Y.F.; data curation, D.C.; investigation, D.B., L.F. and N.E.; software, D.S.; writing—original draft, Y.F.; writing—review and editing, M.V. All authors have read and agreed to the published version of the manuscript.

Funding: This research was funded by the Russian Science Foundation, grant number 19-79-10128.

Acknowledgments: The measurements were partially performed using the equipment of the Joint Center for Collective Use of RTU MIREA.

Conflicts of Interest: The authors declare no conflict of interest.

\section{References}

1. Wang, Y.; Li, J.; Viehland, D. Magnetoelectrics for magnetic sensor application: Status, challenges and perspectives. Mater. Today 2014, 17, 269. [CrossRef]

2. Apicella, V.; Clemente, C.S.; Davino, D.; Leone, D.; Visone, C. Review of modeling and control of magnetostrictive actuators. Actuators 2019, 8, 45. [CrossRef]

3. Gutierrez, J.; Lasheras, A.; Martins, P.; Pereira, N.; Barandiaran, J.M.; Lanceros-Mendez, S. Metallic glass/PVDF magnetoelectric laminates for resonant sensors and actuators: A review. Sensors 2017, 17, 1251. [CrossRef]

4. Palneedi, H.; Annapureddy, V.; Priya, S.; Ryu, J. Status and perspectives of multiferroic magnetoelectric composite materials and applications. Actuators 2016, 5, 9. [CrossRef]

5. Leung, C.M.; Zhuang, X.; Friedrichs, D.; Li, J.; Erickson, R.W.; Laletin, V.; Popov, M.; Srinivasan, G.; Viehland, D. Highly efficient solid state magnetoelectric gyrators. Appl. Phys. Lett. 2017, 111, 122904. [CrossRef]

6. Dong, S.; Li, J.F.; Viehland, D.; Cross, L.E. A strong magnetoelectric voltage gain effect in magnetostrictive-piezoelectric composite. Appl. Phys. Lett. 2004, 85, 3534-3536. [CrossRef]

7. Saveliev, D.; Chashin, D.; Fetisov, L.; Shamonin, M.; Fetisov, Y. Ceramic-heterostructure based magnetoelectric voltage transformer with an adjustable transformation ratio. Materials 2020, 13, 3981. [CrossRef]

8. Srinivasan, G.; Fetisov, Y.K. Microwave magnetoelectric effects and signal processing devices. Integr. Ferroelectr. 2006, 83, 89-98. [CrossRef]

9. Lin, H.; Page, M.R.; McConney, M.; Jones, J.; Howe, B.; Sun, N.X. Integrated magnetoelectric devices: Filters, pico-Tesla magnetometers, and ultracompact acoustic antennas. MRS Bulletin. 2018, 43, 841-847. [CrossRef]

10. Shi, Z.; Wang, C.P.; Liu, X.J.; Nan, C.W. A four-state memory cell based on magnetoelectric composite. Chin. Sci. Bull. 2008, 53, 2135-2138. [CrossRef]

11. Nan, C.-W.; Bichurin, M.I.; Dong, S.; Viehland, D.; Srinivasan, G. Multiferroic magnetoelecrtic composites: Historical perspective, status and future directions. J. Appl. Phys. 2008, 103, 031101. [CrossRef]

12. Vopson, M.M. Fundamentals of multiferroic materials and their possible applications. Crit. Rev. Solid State Mater. Sci. 2014, 40, 1-28. [CrossRef]

13. Bichurin, M.; Filippov, D.; Petrov, V.; Laletsin, V.; Paddubnaya, N.; Srinivasan, G. Resonance magnetoelectric effects in layered magnetostrictive-piezoelectric composites. Phys. Rev. 2003, B68, 132408. [CrossRef]

14. Chashin, D.V.; Fetisov, L.Y.; Saveliev, D.V.; Fetisov, Y.K. Magnetoelectric monolithic resonator based on the ferromagneticpiezoelectric structure excited with a linear current. Sensors Lett. 2019, 3, 2500804. [CrossRef]

15. Josepf, R.I.; Schlomann, E. Demagnetizing field of nonellipsoidal bodies. J. Appl. Phys. 1965, 36, 1579-1593. [CrossRef]

16. Available online: https://metglas.com/magnetic-materials/ (accessed on 3 November 2021).

17. Murthy, S.R.; Rao, T.S. Magnetostriction of Ni-Zn and Co-Zn ferrites. Phys. Stat. Sol. 1985, 90, 631-635. [CrossRef]

18. ELPA Research Institute. Available online: https://www.elpapiezo.ru/ENG/plates_e.html (accessed on 3 November 2021).

19. Vopson, M.M.; Fetisov, Y.K.; Caruntu, G.; Srinivasan, G. Measurement techniques of the magneto-electric coupling in multiferroics. Materials 2017, 10, 963. [CrossRef]

20. Chashin, D.V.; Burdin, D.A.; Fetisov, L.Y.; Ekonomov, N.A.; Fetisov, Y.K. Precise measurements of magnetostriction of ferromagnetic plates. J. Siberian Federal Univ. Math. Phys. 2018, 11, 30-34. [CrossRef]

21. Burdin, D.A.; Saveliev, D.V.; Fetisov, Y.K.; Ekonomov, N.A.; Chashin, D.V. Magnetoelectric structure with integrated current carrying electrodes. J. Phys. Conf. Ser. 2019, 1389, 012049. [CrossRef]

22. Timoshenko, S. Vibration Problems in Engineering; D. Van Nostrand Company, Inc.: Toronto, ON, Canada, $1955 ;$ p. 310.

23. Bichurin, M.I.; Petrov, V.M.; Srinivasan, G. Theory of low-frequency magnetoelectric coupling in magnetostrictive-piezoelectric bilayers. Phys. Rev. B 2003, 68, 054402. [CrossRef]

24. Fetisov, L.Y.; Burdin, D.A.; Ekonomov, N.A.; Chashin, D.V.; Zhang, J.; Srinivasan, G.; Fetisov, Y.K. Nonlinear magnetoelectric effects at high magnetic field amplitudes in composite multiferroics. J. Phys. D Appl. Phys. 2018, 51, 154003. [CrossRef]

25. Chashin, D.V.; Fetisov, L.Y.; Saveliev, D.V.; Fetisov, Y.K. Planar magnetoelectric transformer based on a composite ferromagnetpiezoelectric heterostructure Sens. Actuators A Phys. 2021, 328, 112773. [CrossRef]

26. Fetisov, L.Y.; Fetisov, Y.K.; Perov, N.S.; Chashin, D.V. Magnetoelectric effect in amorphous FeNiSiC ferromagnet-piezoelectric planar structures. Technical Phys. 2011, 56, 485-490. [CrossRef] 
27. Chen, D.X.; Pardo, E.; Sanchez, A. Demagnetizing factors of rectangular prisms and ellipsoids. IEEE Trans. Magn. 1988, 38, 1742-1752. [CrossRef]

28. Fetisov, Y.K.; Chashin, D.V.; Fetisov, L.Y. Magnetoelectric ferrite-piezoelectric heterostructure with coil-free excitation for dc magnetic field sensing. IEEE Sens. Lett. 2021, in press. [CrossRef]

29. Karpenkov, D.Y.; Bogomolov, A.A.; Solnyshkin, A.V.; Karpenkov, A.Y.; Shevyakov, V.I.; Belov, A.N. Multilayered seramic heterostructures of lead zirconate titanate and nickel-zinc ferrite for magnetoelectric sensor elements. Sens. Act. A Phys. 2017, 266, 242. [CrossRef]

30. Pereira, N.; Lima, A.C.; Lanseros-Mendez, S.; Martins, P. Magnetoelectrics: Three centures of research heading towards the 4.0 industrial revolution. Materials 2020, 13, 4033. [CrossRef]

31. Prieto, J.L.; Aroca, C.; Lopez, E.; Sanchez, M.C.; Sanchez, P. Magnetostrictive piezoelectric magnetic sensor with current excitation. J. Magn. Mag. Mater. 2000, 215-216, 756-758. [CrossRef]

32. Burdin, D.A.; Saveliev, D.V.; Ekonomov, N.A.; Chashin, D.V.; Fetisov, Y.K. Effective excitation of magnetoelectric voltage harmonics in a ferromagnet-piezoelectric structure by current passing through the magnetic layer. Technol. Phys. Lett. 2020, 46, 487-490. [CrossRef]

33. Fetisov, L.Y.; Fetisov, Y.K.; Sreenivasulu, G.; Srinivasan, G. Nonlinear resonant magnetoelectric interactions and efficient frequency doubling in a ferromagnetic-ferroelectric layered structure. JAP 2013, 113, 116101. [CrossRef]

34. Burdin, D.A.; Chashin, D.V.; Ekonomov, N.A.; Gordeev, S.N.; Fetisov, Y.K. Nonlinear magnetoelectric effect in a layered ferromagnetic-piezoelectric heterostructure excited by transverse magnetic field. Appl. Phys. Lett. 2020, 116, 072901. [CrossRef]

35. Liang, X.; Dong, C.; Chen, H.; Wang, J.; Wei, Y.; Zaeimbashi, M.; He, Y.; Matyushov, A.; Sun, C.; Sun, N. A review of thin-film magnetoelastic materials for magnetoelectric applications. Sensors 2020, 20, 1532. [CrossRef] [PubMed]

36. Yarar, E.; Hrkac, V.; Zamponi, C.; Piorra, A.; Kienle, L.; Quandt, E. Low temperature aluminum nitride thin films for sensory applications. AIP Adv. 2016, 6, 075115. [CrossRef]

37. Feshenko, V.S.; Zyzblyuk, K.N.; Senokosov, E.A.; Chukita, V.I.; Kiselev, D.A. Features of the receiving of piezoelectric thin films by plasma spraying of powdery AlN. Rus. Technol. J. 2020, 8, 67-79. [CrossRef] 